\title{
Genetic constraint on the evolution of courtship behaviour in Drosophila melanogaster
}

\author{
Mark H. Gromko
}

Department of Biological Sciences, Bowling Green State University, Bowling Green, Ohio 43403, U.S.A.

\begin{abstract}
A quantitative genetic analysis of male courtship behavior in Drosphila melanogaster was carried out. Heritabilities and genetic correlations were calculated from data collected from 330 father-son combinations. Only copulation duration had a heritability significantly different from zero. Copulation duration and fertility were related by a significant positive genetic correlation. A highly significant negative genetic correlation of copulation duration with courtship vigor (towards a virgin female) is possibly responsible for constraining further evolution of copulation duration in this population. It is noteworthy that genetic correlations between traits with low heritabilities were not detectable; practical and theoretical considerations concerning genetic correlations between traits with low heritabilities are discussed.
\end{abstract}

\section{INTRODUCTION}

Directional selection acting consistently over time is expected to produce negative genetic correlations between pairs of adaptively significant traits (Falconer, 1981, p 300; Dickerson, 1955; Robertson, 1955; Rose, 1982). This expectation arises from a consideration of selection acting on pleiotropic genes. Genetic variation for any two fitness characters will be depleted through the fixation of alleles that have positive effects on both characters and through the loss of alleles which have negative effects on both characters. The genetic variation which will remain must therefore be due to alleles at other loci which affect one trait positively and the other negatively. This general formulation applies to any two characters related to fitness.

There is a closely related expectation which specifies the particular classes of characters related through negative genetic correlation. This expectation arises from a consideration of the evolution of senescence in particular and of life history traits in general (Williams, 1957; Gadgil and Bossert, 1970; Rose, 1982). The expectation is for a tradeoff between early and late life history characters. A recent review and evaluation of the data has shown considerable empirical support for this specific expectation (Reznick, 1985; see also Etges, 1982), and progress has been made in the investigation of the physiological basis of this relationship (Service and Rose, 1985).

The more general expectation of negative genetic correlation between any two fitness characters has not received such consistent support. There are several examples of negative correlations in economically important characters in plants and livestock (cf. Antonovics, 1976; Dickerson, 1955; Lande, 1982) and in fitness characters in nondomestic animals as well (Hegmann and Dingle, 1982). But there are also some notable examples where there is no evidence of antagonistic pleiotropy or tradeoff (Mitchell-Olds, 1986; Arnold, 1981).

The general expectation of negative genetic correlation depends upon the assumption of genetic variance arising from overdominant loci. If instead the genetic variance is due to rare recessives, the genetic correlation might be expected to be positive in sign (Falconer, 1981, pp. 306-307). Furthermore, the expectation was derived for characters which are under directional selection. There is no similar unambiguous expectation for the effects of stabilising selection on genetic correlations.

Thus, there is both empirical and theoretical motivation to investigate the genetic correlational structure of characters related to fitness. The 
purpose of the present report is to quantify heritabilities and genetic correlations for seven male characters related to courtship and reproduction in Drosophila melanogaster.

\section{MATERIALS AND METHODS}

The flies used in this experiment originated from a wild population collected at Jeffers Orchard, Grand Rapids, Ohio, in August 1984. A Drosophila melanogaster subpopulation of the JEFFERS collection has been maintained under conditions of minimal inbreeding between forty lines cultured in 8-dram shell vials. For maintenance of this base population, virgins were collected from each of the 40 vials each generation, and crosses were made between males and females from different vials. Each generation the pairings were changed so that parental males and females had minimal common ancestry. Testing of this population began in September 1985, and took 6 months to complete.

Virgin males and females were collected every other week from the stock minimal-inbreeding cultures and were allowed, at 3 to 5 days of age, to mate at random in a small population cage. Virgin males and females to be tested were collected from the one-generation population cage cultures, and were thus random-bred for one generation before testing. All flies were maintained at $25^{\circ} \mathrm{C}$ and were on a 12:12 light cycle throughout the culturing and test period.

All flies were 3-day-old virgins at the time of first testing. One male and one female were aspirated into a plexiglass observation chamber $(3 \mathrm{cc})$ and the following data recorded: latency to initiate courtship (LAGCOURT), time from first courtship to copulation (LAGCOP), copulation duration (COPDUR), and the percentage of time in which the male engaged in any courtship behavior during the interval from the initiation of courtship to the beginning of copulation (VIGOR). If the time from first courtship to copulation was longer than $10 \mathrm{~min}$, VIGOR was assessed in the first $10 \mathrm{~min}$ following the initiation of courtship. Following the completion of copulation, the male was aspirated out and saved in a separate vial for further testing on the following day, as described below. The female was transferred by aspiration five times, staying 2 days in each of the first two vials and 1 day in each of the next three. The number of adult progeny eclosing from the three 1-day cultures (which represent days 4-6 following the first mating) were counted (FERTILITY). In preliminary experiments we found that progeny pro- duction on these days correlated very highly ( $r>$ 0.95 ) with total progeny production. On the day after the courtship and mating between virgin flies, each male was paired in the plexiglass observation chamber with a female that had mated the previous day with a different male. For these 4-day-old nonvirgin flies, the latency to initiate courtship (2ND LAGCOURT) and the percentage of time spent in courtship in the first 10 minutes following the initiation of courtship (2ND VIGOR) were recorded. The experimental design is summarised in fig. 1.

A son of each male was collected as a virgin and the same data as above were recorded for sons paired with females from the random-bred base population cage. In order to standardise culture conditions among sons of different mothers, sons were collected from one of the two 2-day cultures since there was much less variability in females' productivity in the first few days following copulation than there was in later days. Thus sons from mothers of different fecundity were collected from vials of similar larval density. Data from 330 father-son combinations are reported here.

To render distributions normal, LAGCOURT, LAGCOP, and 2ND LAGCOURT were transformed to natural logs, and VIGOR and 2ND VIGOR were transformed to arcsines. COPDUR and FERTILITY did not require transformation.

Narrow-sense heritabilities and their standard errors were estimated from offspring-parent regression (Falconer, 1981):

$$
\begin{gathered}
h^{2}=2 b_{x^{\prime} x} \\
\text { S.E. }\left(h^{2}\right)=\frac{2}{\sqrt{N}} \quad(\text { approx })
\end{gathered}
$$

where $x^{\prime}$ and $x$ are the values of the trait in offspring and parents, respectively. Genetic correlations were calculated following VanVleck and Henderson (1961) and standard errors according to Falconer (1981) and Robertson (1959):

$$
\begin{gathered}
r_{A}=\frac{\frac{1}{2}\left(\operatorname{cov} x y^{\prime}+\operatorname{cov} x^{\prime} y\right)}{\sqrt{\left(\operatorname{cov} x x^{\prime}\right)\left(\operatorname{cov} y y^{\prime}\right)}} \\
\text { S.E. }\left(r_{A}\right)=\frac{1-r_{A}^{2}}{\sqrt{2}} \times \sqrt{\frac{\text { S.E. }\left(h_{x}^{2}\right) \times \text { S.E. }\left(h_{y}^{2}\right)}{h_{x}^{2} \times h_{y}^{2}}}
\end{gathered}
$$

where $y^{\prime}$ and $y$ represent the values of a second trait in offspring and parents.

Environmental correlations, $r_{E}$, were calculated from

$$
r_{p}=h_{x} h_{y} r_{A}+e_{x} e_{y} r_{E}
$$

where $e^{2}=1-h^{2}$ (Falconer, 1981). 


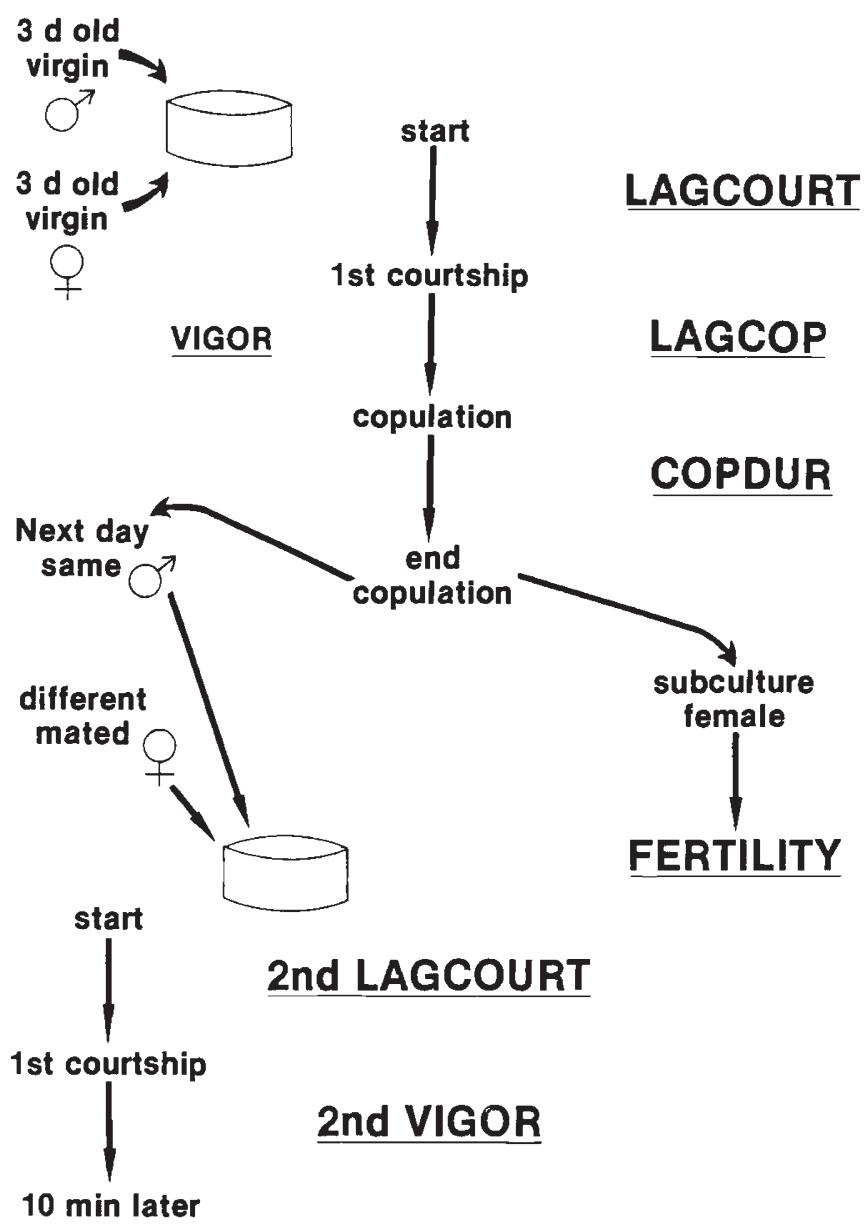

Figure 1 Summary of experimental design.

Principal component analysis was used to describe the pattern of phenotypic and environmental variation among fathers. This, and all statistical analysis, was executed using SAS (SAS Institute Inc. 1985).

\section{RESULTS}

Means and 95 per cent confidence intervals were calculated on the normalised data; these data are shown backtransformed to the original scale of measurement in table 1. Fathers' and sons' copulation durations are significantly different $(p<$ $0.0001)$; all other comparisons are not significantly different. The similarity of fathers' and sons' scores for six of the seven variables argues against the existence of any differences in fathers' and sons' environments affecting the values of these variables. If differences in fathers' and sons' environ- ments have affected copulation duration, the heritability of COPDUR is probably larger than the estimate obtained from these data.

The matrix of phenotypic correlations among fathers' scores is shown in table 2 . The pattern of covariation in this matrix was explored with principal component analysis, the results of which are shown in table 3 . The first three principal components (PC's) each had eigenvalues greater than 1.0 and together explained 58 per cent of the variation in the data set. The variables VIGOR and LAGCOP load heavily on the first PC. The loadings are opposite in sign, making this $\mathrm{PC}$ a consistent representation of courtship towards a virgin female, since high VIGOR and short LAGCOP would both be expected to contribute to fitness. Similarly, the variables 2ND LAGCOURT and 2ND VIGOR both load strongly, with opposite sign, on PC2, which thus represents courtship towards a mated female. The variables COPDUR 
Table 1 Means and 95 per cent confidence intervals calculated on transformed data and backtransformed to original scale of measurement

\begin{tabular}{|c|c|c|c|}
\hline Variable & $L_{1}$ & Mean & $L_{2}$ \\
\hline \multicolumn{4}{|c|}{ LAGCOURT (min) } \\
\hline fathers & 0.98 & $1 \cdot 10$ & $1 \cdot 22$ \\
\hline sons & $1 \cdot 05$ & $1 \cdot 18$ & $1 \cdot 33$ \\
\hline \multicolumn{4}{|c|}{ VIGOR (\%) } \\
\hline fathers & $81 \cdot 58$ & $84 \cdot 53$ & $87 \cdot 27$ \\
\hline sons & $79 \cdot 42$ & $82 \cdot 48$ & $85 \cdot 35$ \\
\hline \multicolumn{4}{|c|}{ LAGCOP (min) } \\
\hline fathers & $1 \cdot 82$ & $2 \cdot 10$ & $2 \cdot 45$ \\
\hline sons & $2 \cdot 12$ & $2 \cdot 47$ & $2 \cdot 88$ \\
\hline \multicolumn{4}{|c|}{ 2ND LAGCOURT (min) } \\
\hline fathers & $1 \cdot 10$ & $1 \cdot 23$ & $1 \cdot 38$ \\
\hline sons & $1 \cdot 15$ & $1 \cdot 27$ & $1 \cdot 42$ \\
\hline \multicolumn{4}{|c|}{ 2ND VIGOR $(\%)$} \\
\hline fathers & $20 \cdot 74$ & $23 \cdot 44$ & $26 \cdot 25$ \\
\hline sons & $18 \cdot 62$ & $21 \cdot 12$ & $23 \cdot 74$ \\
\hline \multicolumn{4}{|c|}{ COPDUR (min) } \\
\hline fathers & $19 \cdot 37$ & $19 \cdot 87$ & $20 \cdot 37$ \\
\hline sons & $17 \cdot 25$ & $17 \cdot 73$ & $18 \cdot 20$ \\
\hline \multicolumn{4}{|c|}{ FERTILITY (count) } \\
\hline fathers & $133 \cdot 4$ & $140 \cdot 2$ & $146 \cdot 9$ \\
\hline sons & $133 \cdot 7$ & $140 \cdot 7$ & $147 \cdot 7$ \\
\hline
\end{tabular}

and FERTILITY load highly on PC3, which represents copulation and sperm transfer. The variable LAGCOURT loaded fairly evenly on all three components. Because this analysis was done without rotation, these PC's represent relatively independent patterns of variation; for instance, a male's courtship activity towards a mated female was relatively independent of his courtship activity towards a virgin.

Heritabilities are shown on the diagonal in table 4, and genetic correlations above the diagonal The standard error of the heritability estimates is $0 \cdot 11$. The estimate of heritability for COPDUR is greater than 4 times its standard error; none of the other estimates approach statistical significance. Many of the estimates of genetic correlation were much greater than 1.0 or much less than -1.0 and had very large standard errors. In all cases where estimates of $r_{A}$ were less than their standard error, indicating a genetic correlation not different from zero, no value is reported. The genetic correlation between VIGOR and COPDUR is negative and greater than six times its standard error, while that between COPDUR and FERTILITY is positive and more than twice its standard error. The remaining two genetic correlations have large standard errors and are not significantly different from zero despite their apparent large values. Also note for these two correlations that the effect on fitness is opposite to the sign of the correlation. For instance, high values of VIGOR would contribute to fitness but high values of LAGCOP would not, so the positive correlation between these values reflects an antagonistic interaction in terms of fitness. Because of all the zero correlations in this matrix, the matrix itself represents independent patterns of variation, and there is no need to (in fact, it is not possible to) factor this genetic correlation matrix.

For the purpose of calculating environmental correlations, the two genetic correlations in table 4 which are greater or less than 1 or -1 , respectively, had one standard error subtracted or added, so that their values fell between 1 and -1 . Environmental correlations are shown in table 5. Principal component analysis was carried out on this matrix, yielding four factors with eigenvalues greater than one, which together explained 73 per cent of the variation in the data set. The factor pattern, shown in table 6 , is very similar to that of the matrix of phenotypic correlations. The first PC represents courtship towards virgin females, and PC2 represents courtship towards nonvirgin females. Finally, COPDUR and FERTILITY here appear to be somewhat more independent than in the phenotypic analysis, with COPDUR loading more on PC3 and fertility more on PC4.

Table 2 Matrix of phenotypic correlations $\left(r_{p}\right)$ among fathers' characters

\begin{tabular}{|c|c|c|c|c|c|c|c|}
\hline & LAGCOURT & VIGOR & LAGCOP & $\begin{array}{l}\text { 2ND } \\
\text { LAGCOURT }\end{array}$ & $\begin{array}{l}\text { 2ND } \\
\text { VIGOR }\end{array}$ & COPDUR & FERTILITY \\
\hline $\begin{array}{l}\text { LAGCOURT } \\
\text { VIGOR } \\
\text { LAGCOP } \\
\text { 2ND LAGCOURT } \\
\text { 2ND VIGOR } \\
\text { COPDUR } \\
\text { FERTILITY }\end{array}$ & & 0.00 & $\begin{array}{l}-0 \cdot 17^{* *} \\
-0 \cdot 46^{* * *}\end{array}$ & $\begin{array}{c}0.15^{* *} \\
0.10 \\
-0.03\end{array}$ & $\begin{array}{l}-0 \cdot 14^{* *} \\
0 \cdot 11^{*} \\
-0 \cdot 07 \\
-0 \cdot 21^{* * *}\end{array}$ & $\begin{array}{r}0.00 \\
-0.03 \\
0.04 \\
-0.04 \\
0.01\end{array}$ & $\begin{array}{l}0.01 \\
-0.06 \\
0.03 \\
-0.09 \\
0.10 \\
0.15^{* *}\end{array}$ \\
\hline
\end{tabular}

\footnotetext{
$* p<0.05$.

$* * p<0.01$.

$* * * p<0.001$.
} 
Table 3 Principal component analysis on the matrix of phenotypic correlations presented in table 2

\begin{tabular}{lrrr}
\hline & PC1 & \multicolumn{1}{c}{ PC2 } & \multicolumn{1}{c}{ PC3 } \\
\hline LAGCOURT & 0.3435 & 0.3919 & 0.5006 \\
VIGOR & 0.7810 & 0.3165 & -0.0473 \\
LAGCOP & -0.7997 & -0.2475 & -0.1613 \\
2ND LAGCOURT & 0.3225 & -0.5958 & 0.0837 \\
2ND VIGOR & -0.0041 & 0.7486 & -0.1094 \\
COPDUR & -0.1947 & 0.1436 & 0.6845 \\
FERTILITY & -0.2597 & 0.3362 & 0.6090 \\
\hline
\end{tabular}

\section{DISCUSSION}

The pattern of genetic variation and covariation revealed in table 4 demonstrates the existence of genetic constraint on the evolution of male courtship characters. The variable COPDUR has sufficiently high heritability that it might be expected to respond to selection, either because it is itself a fitness character or because of its positive genetic correlation with FERTILITY. The existence of a large negative genetic correlation of COPDUR with VIGOR might be constraining further evolution of copulation duration.

The pattern of covariation in phenotypes (table 4 ) is very similar to the pattern of covariation due to environment (table 6). There was no consistent relationship between the courtship and reproductive characters measured and the environmental variables temperature, relative humidity and barometric pressure (data not shown). The next most obvious component of the males' environment that might be responsible for the patterns of covariation observed is the female. In this interpre- tation, that the virgin female with which the male was paired was either more or less attractive provides the dimensions of $\mathrm{PC} 1$, and likewise for the nonvirgin female with which he was paired the following day (PC2). Since the two females with which a male was paired were different individuals, it is reasonable that their degree of attractiveness should be relatively independent. That CODPUR and FERTILITY loaded together on PC3 in the analysis of phenotypes is probably due to the positive genetic correlation between them. In contrast, these two variables tended to load more independently in the analysis of environmental correlations, suggesting that they have little common environmental source of variation.

Some formulations are explicit in expecting negative genetic correlations in the context of low heritabilities (Robertson, 1955). Others suggest that negative genetic correlation and large heritabilities should be coupled (Rose, 1982). Practically speaking, the form the equation for the genetic correlation (2) and for its standard error (4) suggests that it will be difficult to demonstrate significant genetic correlations for traits with low heritabilities (Robertson, 1959; VanVleck and Henderson, 1961). The standard error involves the product of two heritabilities in the denominator, so as the heritabilities get small, the standard error gets very large. The genetic correlation itself also involves the product of two covariances in the denominator, making the estimate shift unreliably for traits with low heritability (Mitchell-Olds, 1986). In the present study, standard errors for genetic correlations between traits with small

Table 4 Matrix of heritabilities (on diagonal) and genetic correlations ( $r_{A}$, above diagonal). The S.E.s of genetic correlations are shown in parentheses, and the sign of the correlation, in terms of its effect on fitness is shown below that. No value is reported for any genetic correlation which was smaller than its standard error

\begin{tabular}{|c|c|c|c|c|c|c|c|}
\hline & LAGCOURT & VIGOR & LAGCOP & $\begin{array}{l}\text { 2ND } \\
\text { LAGCOURT }\end{array}$ & $\begin{array}{l}2 \mathrm{ND} \\
\text { VIGOR }\end{array}$ & COPDUR & FERTILITY \\
\hline LAGCOURT & 0.01 & - & - & - & - & - & - \\
\hline VIGOR & & 0.09 & $\begin{array}{c}1.33 \\
(-0.74) \\
(-)\end{array}$ & - & - & $\begin{array}{c}-0.81 \\
(0.13) \\
(-)\end{array}$ & - \\
\hline LAGCOP & & & 0.07 & - & - & - & - \\
\hline 2ND LAGCOURT & & & & $0 \cdot 01$ & - & - & $\begin{array}{c}-1 \cdot 16 \\
(-0.76) \\
(+)\end{array}$ \\
\hline 2ND VIGOR & & & & & $0 \cdot 06$ & - & - \\
\hline COPDUR & & & & & & $0 \cdot 46$ & $\begin{array}{c}0.55 \\
(0 \cdot 20) \\
(+)\end{array}$ \\
\hline FERTILITY & & & & & & & $0 \cdot 15$ \\
\hline
\end{tabular}


Table 5 Matrix of environmental correlations $\left(r_{E}\right)$

\begin{tabular}{|c|c|c|c|c|c|c|c|}
\hline & LAGCOURT & VIGOR & LAGCOP & $\begin{array}{l}\text { 2ND } \\
\text { LAGCOURT }\end{array}$ & $\begin{array}{l}\text { 2ND } \\
\text { VIGOR }\end{array}$ & COPDUR & FERTILITY \\
\hline LAGCOURT & & 0.01 & -0.18 & 0.15 & -0.15 & -0.01 & 0.01 \\
\hline VIGOR & & & -0.55 & $0 \cdot 10$ & 0.12 & 0.25 & -0.07 \\
\hline LAGCOP & & & & -0.03 & -0.08 & 0.06 & 0.03 \\
\hline 2ND LAGCOURT & & & & & $-0 \cdot 22$ & -0.06 & -0.08 \\
\hline 2ND VIGOR & & & & & & 0.01 & $0 \cdot 11$ \\
\hline COPDUR & & & & & & & 0.01 \\
\hline FERTILITY & & & & & & & \\
\hline
\end{tabular}

Table 6 Principal component analysis on the matrix of environmental correlations presented in table 5

\begin{tabular}{lrrrr}
\hline & PC1 & \multicolumn{1}{c}{ PC2 } & \multicolumn{1}{c}{ PC3 } & \multicolumn{1}{c}{ PC4 } \\
\hline LAGCOURT & 0.2540 & -0.5187 & -0.3180 & 0.4424 \\
VIGOR & 0.8749 & 0.1533 & 0.1544 & -0.0318 \\
LAGCOP & -0.8288 & -0.0127 & 0.3304 & 0.1268 \\
2ND LAGCOURT & 0.1845 & -0.6618 & 0.0690 & 0.0136 \\
2ND VIGOR & 0.1262 & 0.7148 & -0.2546 & -0.1292 \\
COPDUR & 0.2421 & 0.2378 & 0.7504 & 0.5166 \\
FERTILITY & 0.1278 & 0.3021 & -0.4477 & 0.7099 \\
\hline
\end{tabular}

heritabilities were sometimes as much as two orders of magnitude greater than the estimates of the correlation. Mitchell-Olds (1986) reported a similar situation wherein low heritabilities made estimation of genetical correlation impractical.

It seems clear that the matrix of genetic correlations can and does evolve (Lofsvold, 1986; Sheridan and Barker, 1974; Nordskog, 1977; Mitchell-Olds and Rutledge, 1986). In what sense, then, could the existence of genetic correlations provide constraints on the long-term evolution of characters related to fitness? Genetic correlations that are due to linkage are clearly expected to be transitory; the possibility that pleiotropy itself may evolve should receive consideration. Pleiotropic effects of a single allele might evolve independently through the effects of modifier genes which influence the pleiotropic effects separately. If a pair of traits were both consistently subject to selection, constraints arising early in an evolutionary history might slowly be lessened through selection on such modifier genes. The end state for characters closely related to fitness would be low heritability and low genetic correlation. Only in the case of traits not consistently selected or not closely related to fitness, as is perhaps the case for CODPUR in this study, would selection be insufficient to overcome constraints imposed by negative genetic correlation. In the case of the specific prediction of negative genetic correlation between early and late life-history traits there may be some additional "inherently physiological" (Service et al., 1985) basis for the tradeoff (competing demands on finite resources) that could not be overcome in this way (Gadgil and Bossert, 1970).

Of course, if the behaviours measured in this study do not have substantial impact on fitness then we would not expect negative genetic correlations among them. A straightforward case can be made for six of the seven characters studied; the status of copulation duration as a fitness character is, however, arguable. Perhaps variation in copulation duration above some minimum required for sperm transfer might have no impact on fitness. Alternatively, if copulation were a hazardous activity (due, for instance, to increased risk of predation) or if long copulations were merely wasteful of time, then copulation duration might be under stabilising selection. Another caveat regarding the interpretation of these data is that the measured behaviours represent age-specific samples rather than life-time values of fitnessrelated activities. Resolution of these questions must await further study, and the current interpretation couched in terms of the assumption that the behaviors measured are adequate measures of fitness.

A final point for consideration is the origin and history of the population to be assayed for genetic correlations. Service and Rose (1985) have shown that introducing organisms into a novel environment has the effect of making genetic correlations 
less negative than they were in the familiar environment. They therefore advocate assaying organisms in the environment to which they are adapted. It also appears important to avoid the use of inbred lines, as inbreeding too can shift genetic correlations in a positive direction (Rose, 1984). Hegman and Dingle (1982) emphasize the need to sample organisms from different environments so as to undertake a comparative study. The importance of assessing genotype-by-environment interactions and local adaptation is clear (Via, 1984; Mitchell-Olds, 1986). The objective of assaying these parameters as they exist in natural populations is thus somewhat at odds with the need to have the population adapted to the laboratory environment in which it is to be tested. The population assayed in this report was kept in the laboratory for 1 year before testing began, and so has had opportunity to adapt to the laboratory environment. The minimal inbreeding procedures represent an attempt to maintain laboratory cultures under constant conditions that create minimal selective pressures. The goal of this method of maintaining a stock was to have the laboratory environment present minimal adaptive challenges to the population to be assayed, and in this way to allow local population attributes to be perceived.

Acknowledgements I thank K. Carr for technical assistance; and M. E. Newport, P. Service, E. Speiss, and K. Vessey for criticism of the manuscript. This work was supported by NSF Grant BNS-84i8934.

\section{REFERENCES}

ANTONOVICS, J. 1976. The nature of limits to natural selection. Ann. Missouri Bot. Gard., 63, 224-247.

ARNOLD, S. J. 1981. Behavioural variation in natural populations. I. Phenotypic, genetic and environmental correlations between chemoreceptive responses to prey in the garter snake, Thamnophis elegans. Evolution, 35, 489-509.

DICKERSON, G. E. 1955. Genetic slippage in response to selection for multiple objectives. CSHSQB, 20, 213-224.

ETGES, W. J. 1982. "A new view of life-history evolution"?-A response. Oikos, 38, 118-122.

FALCONER, D. S. 1981. Introduction to Quantitative Genetics, 2nd edition. Longman, London.

GADGIL, M. AND BOSSERT, w. 1970. Life historical consequences of natural selection. Am. Nat., 104, 1-24.
HEGMANN, J. P. AND DINGLE, H. 1982. Phenotypic and genetic covariance structure in milkweek bug/life history traits. In Dingle, H. and Hegmann, J. P. (eds.), Evolution and Genetics of Life Histories, Springer-Verlag, New York, pp. 177-185.

ISTOCK, C. A. 1983. The extent and consequences of heritable variation for fitness characters. In King, C. E. and Dawson, P. S. (eds.), Population Biology Retrospect and Prospect, Columbia University Press, New York, pp. 61-96.

LANDE, R. 1982. A quantitative genetic theory of life history evolution. Ecology, 63, 607-615.

LOFSVOLD, D. 1986. Quantitative genetics of morphological differentation in Peromyscus. I. Tests of the homogeneity of genetic covariance structure among species and subspecies. Evolution, 40, 559-573.

MITCHELL-OLDS, T. AND RUTLEDGE, J. J. 1986. Quantitative genetics in natural plant populations: A review of the theory. Am. Nat., 127, 379-402.

NORDSKOG, A. W. 1977. Success and failure of quantitative genetic theory in poultry. In Pollak, E., Kempthorne, O. and Bailey, T. B. Jr. (eds.) Proceedings of the International Conference on Quantitative Genetics, The Iowa State University Press, Ames, pp. 569-586.

REZNICK, D. 1985. Costs of reproduction: an evaluation of the empirical evidence. Oikos, 44, 257-267.

ROBERTSON, A. 1955. Selection in animals: Synthesis. CSHSQB, 20, 225-229.

ROBERTSON, A. 1959. The sampling variance of the genetic correlation coefficient. Biometrics, 15, 469-485.

ROSE, M. R. 1982. Antagonistic pleiotropy, dominance, and genetic variation. Heredity, 48, 63-78.

ROSE, M. R. 1984. Genetic covariation in Drosophila life history: untangling the data. Am. Nat., 123, 565-569.

SAS INSTITUTE INC. 1985. SAS User's Guide: Basics, Version 5 Edition. Cary, NC: SAS Institute Inc.

SAS INSTITUTE INC. 1985. SAS User's Guide: Statistics, Version 5 Edition. Cary, NC: SAS Institute Inc.

SERVICE, P. M. AND ROSE, M. R. 1985. Genetic covariation among life-history components: the effect of novel environments. Evolution, 39, 943-945.

SERVICE, P. M., HUTCHINSON, E. W., MACKINLEY, M. D. AND ROSE, M. R. 1985. Resistance to environmental stress in Drosophila melänogaster selected for postponed senescence. Physiol. Zool., 58, 380-389.

SHERIDAN, A. K. AND BARKER, J. S. F. 1974. Two-trait selection and the genetic correlation. II. Changes in the genetic correlation during two-trait selection. Aust. J. Biol. Sci., 27, 89-101.

VANVLECK, L. D. AND HENDERSON, C. R. 1961. Empirical sampling estimates of genetic correlations. Biometrics, 17, 359-371.

VIA, S. 1984. The quantitative genetics of polyphagy in an insect herbivore. I. Genotype-environment interaction in larval performance on different host plant species. Evolution, 38, 881-895.

WILliAMS, G. C. 1957. Pleiotropy, natural selection, and the evolution of senescence. Evolution, 11, 398-411. 